\title{
Polyamines Promote the Biosynthesis of Ethylene in Detached Rice Leaves
}

\author{
Sheng Lee Chen, Chien Teh Chen and Ching Huei Kao
}

\author{
Department of Agronomy, National Taiwan University, Taipei, Taiwan, Republic of China
}

\begin{abstract}
The effects of polyamines (putrescine, spermidine, spermine and diaminopropane) on the production of ethylene in detached rice leaves were investigated. Polyamines effectively promoted the production of ethylene in detached rice leaves under both light and dark conditions. Putrescine stimulated the production of ethylene within 4 hours of its application, a result suggests that putrescine enhances the production of ethylene directly. Putrescine also stimulated the production of ethylene in detached leaves that had been aged for 2 and 4 days. The stimulatory effect of putrescine resulted from the enhancement of the synthesis of 1-aminocyclopropane-1carboxylic acid (ACC) and the conversion of ACC to ethylene. . The activity of S-adenosylmethionine decarboxylase in segments of rice leaves was inhibited by the application of putrescine. Thus, the enhancement of the synthesis of ACC by putrescine seems to be mediated by increases in the activity of ACC synthase and in the level of the substrate (S-adenosylmethionine) for ACC synthase.
\end{abstract}

Key words: S-Adenosylmethionine - Ethylene production - Putrescine - Spermidine - Spermine - Oryza sativa.

In a recent review, Evans and Malmberg (1989) cited several studies that demonstrated that polyamines inhibit both the production of ethylene and senescence. The mechanism of retardation of leaf senescence by exogenous polyamines has been proposed to be related to their inhibition of the biosynthesis of ethylene (Fuhrer et al. 1982, Shih et al. 1982). We also found some time ago that polyamines retard the senescence of detached rice leaves (Cheng and Kao 1983). In order to understand whether the mechanism of the retardation of senescence of detached rice leaves by polyamines is related to the inhibition of the biosynthesis of ethylene by these compounds, we studied the effects of polyamines, specifically putrescine, on the biosynthesis of ethylene. Unexpectedly, we found that polyamines stimulated the production of ethylene via enhancement of the synthesis of ACC and the conversion of ACC to ethylene.

\section{Materials and Methods}

Seedlings of rice (Oryza sativa cv. Taichung Native 1)

Abbreviations: ACC, 1-aminocyclopropane-1-carboxylic acid; Dap, diaminopropane; Put, putrescine; SAM, S-adenosylmethionine; SAMDC, S-adenosylmethionine decarboxylase; Spd, spermidine; Spm, spermine. were grown in hydroponic culture as described previously (Chen et al. 1990). The apical $3 \mathrm{~cm}$ of the third leaves of 12-day-old seedlings were used for the experiments. Ten segments of rice leaves, weighing about $45 \mathrm{mg}$, were floated on $10 \mathrm{ml}$ of test solution in a Petri dish. All samples were kept at $27^{\circ} \mathrm{C}$ in the dark.

After various periods of incubation, the leaf samples were transferred to a 14-ml test tube. The tubes were sealed with serum caps. After $1 \mathrm{~h}$ of incubation at $27^{\circ} \mathrm{C}$ in the dark, a 1-ml gas sample was withdrawn from the headspace of each test tube. Ethylene was assayed with a gas chromatograph equipped with an alumina column and a flame ionization detector. Absolute levels of ethylene varied among experiments because of seasonal effects. However, the patterns of responses to polyamines were reproducible.

ACC was extracted and quantitated as described previously (Kao and Yang 1982).

For the assay of SAMDC activity, leaf segments were homogenized in $25 \mathrm{~mm}$ sodium phosphate $(\mathrm{pH} 7.5)$ that contained $0.1 \mathrm{~mm}$ dithiothreitol, $1 \mathrm{~mm}$ pyridoxal-5-phosphate and $20 \mathrm{mM} \mathrm{Na}$-EDTA, and centrifuged at $17,000 \times \mathrm{g}$ for $20 \mathrm{~min}$ at $4^{\circ} \mathrm{C}$. The supernatant was used for the assay of enzymatic activity, which involved measuring the evolution of ${ }^{14} \mathrm{CO}_{2}$ from SAM- $\left[{ }^{14} \mathrm{C}\right]$ carboxyl $\left(55 \mathrm{mCi} \mathrm{mmol}^{-1}\right.$, Amersham), as described by Kaur-Sawhney et al. (1982). Protein content was measured by the method of Lowry et 
al. (1951).

All experiments were repeated at least three times. Similar results and identical trends were obtained on each occasion. The data reported here are all from a single experiment.

\section{Results}

The effects of the concentration of Dap, Put, Spd and Spm on the production of ethylene in detached rice leaves in darkness are presented in Fig. 1. Dap, Put, Spd and Spm each significantly promoted the production of ethylene in detached rice leaves. Increasing concentrations of Spd or Spm from 1 to $10 \mathrm{~mm}$ progressively enhanced the production of ethylene by detached rice leaves. However, Dap, a product of the oxidation of naturally occurring polyamines, and Put at $5 \mathrm{~mm}$ caused maximum rates of production of ethylene, and no further increase in rates of production could be detected when this concentration was increased to $10 \mathrm{~mm}$. The ability of Dap, Put, Spd and Spm to stimulate the production of ethylene was also observed under light conditions (data not shown). Since all polyamines tested were effective in stimulating the production of ethylene in detached rice leaves, Put was chosen for fur-

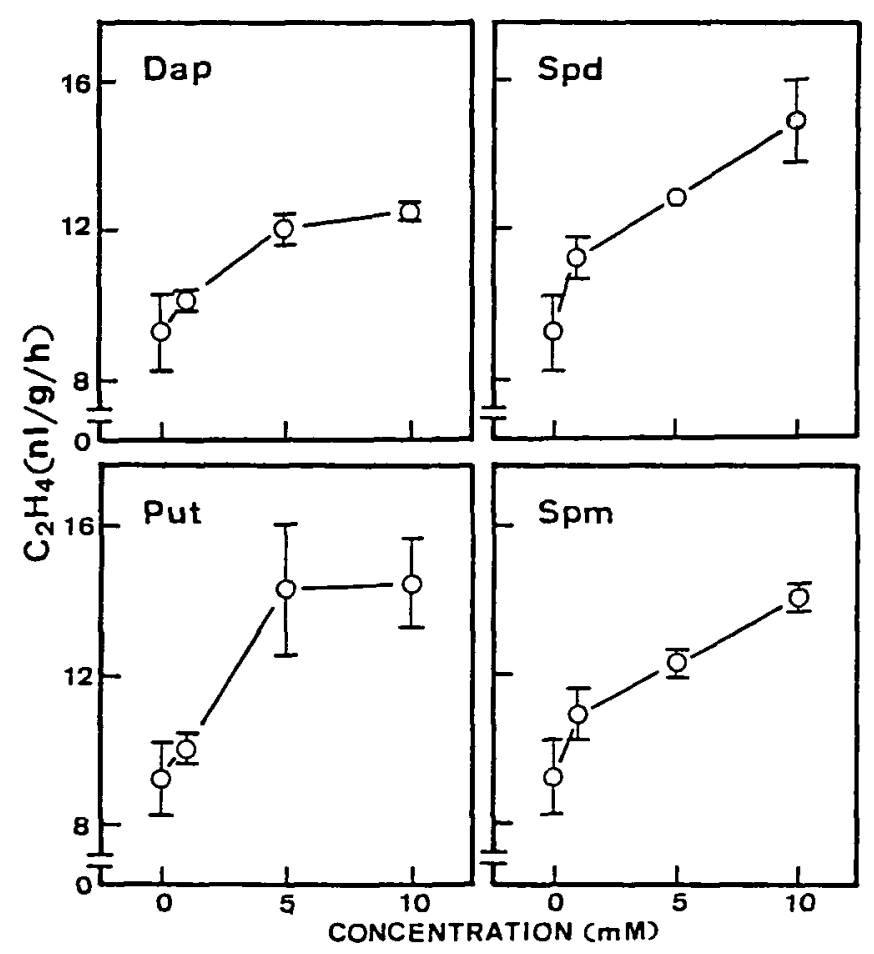

Fig. 1 Relationship between concentration of diaminopropane (Dap), putrescine (Put), spermidine (Spd) and spermine (Spm) and the production of ethylene in segments of rice leaves. The production of ethylene was assayed after $6 \mathrm{~h}$ of incubation in darkness. Bars indicate standard errors. ther detailed studies on the effects of polyamine on the production of ethylene.

Figure 2 shows the changes with time in rates of the production of ethylene in detached rice leaves treated with $10 \mathrm{~mm}$ Put. Promotion of the production of ethylene by Put was detected at $4 \mathrm{~h}$ after the start of incubation in the dark. Increased duration of treatment with Put increased the production of ethylene by detached rice leaves. Incubation periods longer than $8 \mathrm{~h}$ did not result in higher rates of production of ethylene.

The rate of production of ethylene is generally controlled by the level of ACC, the immediate precursor of ethylene (Yang and Hoffman 1984). The effects of Put on the level of ACC were examined and the results are shown in Figure 2. In control leaves, levels of ACC increased slightly within 8 and $12 \mathrm{~h}$ of the start of the incubation. Treatment with Put resulted in higher levels of ACC than those in the controls at 8 and $12 \mathrm{~h}$ after incubation. These results suggest that an enhanced rate of production of ethylene in Put-treated segments of rice leaves is regulated by the promotion of the synthesis of ACC. Since no increase in the level of ACC but an increase in production of
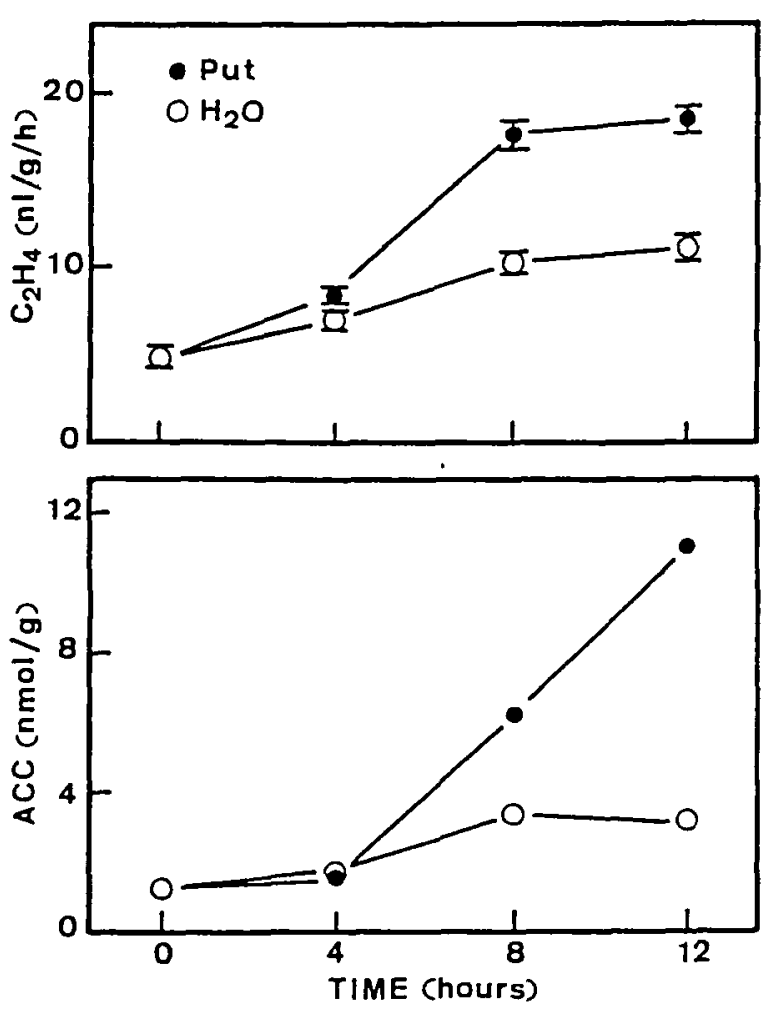

Fig. 2 Changes with time in rates of production of ethylene (upper) and levels of ACC (lower) in segments of rice leaves treated with Put. Segments were treated with either water or $10 \mathrm{~mm}$ Put in darkness. Production of ethylene and levels of ACC were quantitated at the times indicated. 
ethylene was observed after $4 \mathrm{~h}$ of incubation in the Put, the possibility that the conversion of ACC to ethylene is promoted by Put cannot be ruled out. This possibility was tested by measuring the production of ethylene in the presence of a saturating concentration of $A C C(10 \mathrm{mM})$. As indicated in Figure 3, the conversion of $A C C$ to ethylene was promoted by Put during a $12-\mathrm{h}$ incubation.

Both ethylene and polyamines share SAM as a common precursor (Miyazaki and Yang 1987). The observed increase in levels of ACC induced by Put can be explained by an increase in the activity of ACC synthase, by an increase in the level of the substrate (SAM) for ACC synthase, or by both mechanisms. For reasons not yet understood, the activity of ACC synthase in homogenates of segments of rice leaves is undetectable, as is also the case for ACC synthase from internodes of deep-water rice plants (Metraux and Kende 1984). If the increase in the level of ACC indeed by Put is mediated by an increase in levels of SAM, then the activity of SAMDC in rice leaves would be expected to be inhibited by treatment with Put. As indicated in Figure 4, such inhibition does indeed occur. The difference in levels of ACC, $12 \mathrm{~h}$ after the start of incubation, between the control and Put-treated samples was large but that between the activities of SAMDC in control and Put-treated samples was rather small (Figs. 2 and 4). Thus, the possibility that the activity of ACC synthase was increased by Put cannot be ruled out.

To study the effects of aging, segments of rice leaves were incubated in water in darkness for 2 or 4 days before the application of Put. Segments of leaves aged for 2 or 4 days all demonstrated the Put-induced production of ethylene (Fig. 5). However, detached rice leaves aged for 4 days were less responsive to Put in terms of the produc-

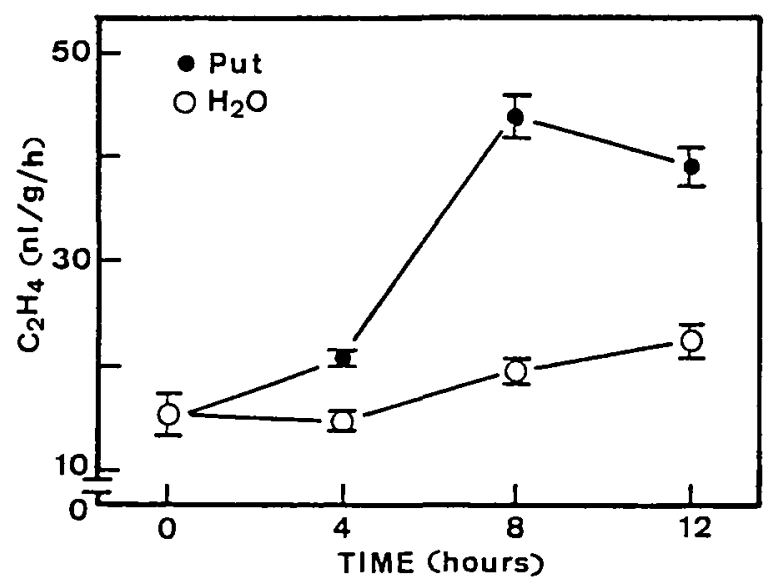

Fig. 3 Effects of Put on the conversion of ACC to ethylene. Segments of rice leaves were pretreated with a saturating concentration of $A C C(10 \mathrm{mM})$ for $2 \mathrm{~h}$ and then treated with either water or $10 \mathrm{mM}$ Put in darkness. Rates of production of ethylene were determined at the times indicated. Bars indicate standard errors.

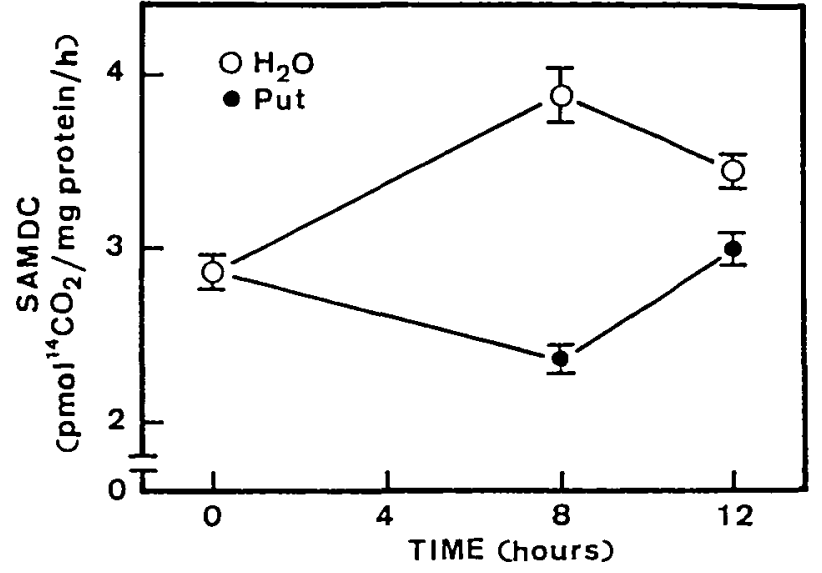

Fig. 4 Effects of Put on the activity of SAMDC in segments of rice leaves. Segments were treated with water or with $10 \mathrm{~mm}$ Put in darkness. The activity of SAMDC was assayed at the times indicated. Bars indicate standard errors.

tion of ethylene. Untreated segments of leaves produced low levels of ethylene regardless of the duration of aging.

The increase in rates of production of ethylene in segments of rice leaves is not specific for the rice cultivar used in this study. The production of ethylene was also promoted by Put in detached leaves of five other cultivars of rice (Fig. 6).

Since tobacco leaf discs were employed by Apelbaum et al. (1981) in their studies of the effects of polyamines on the ACC-induced production of ethylene, we also exam-

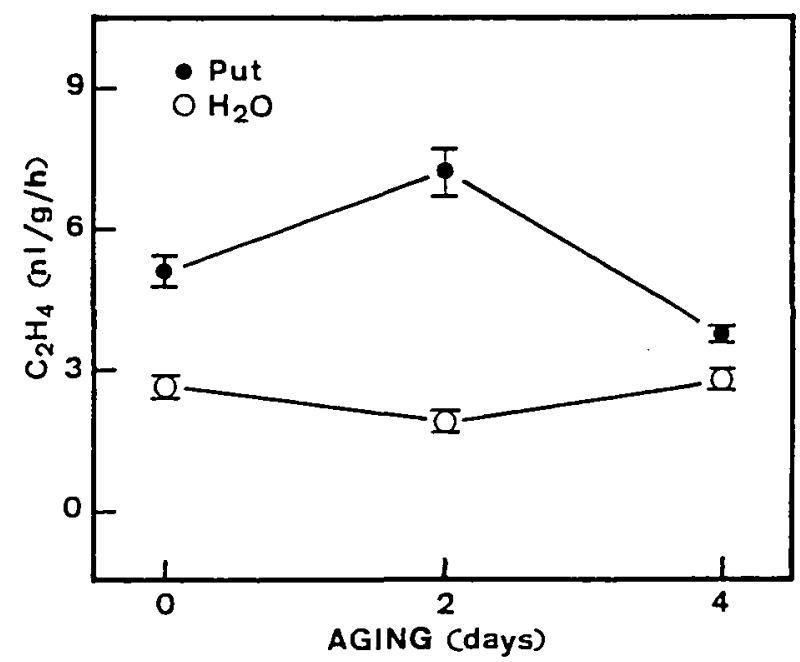

Fig. 5 Effects of aging on the Put-induced production of ethylene in segments of rice leaves. Segments were aged for 2 and 4 days in water and then treated with either water or $10 \mathrm{~mm}$ Put in darkness. The production of ethylene was assayed $6 \mathrm{~h}$ after the initiation of treatment. Bars indicate standard errors. 


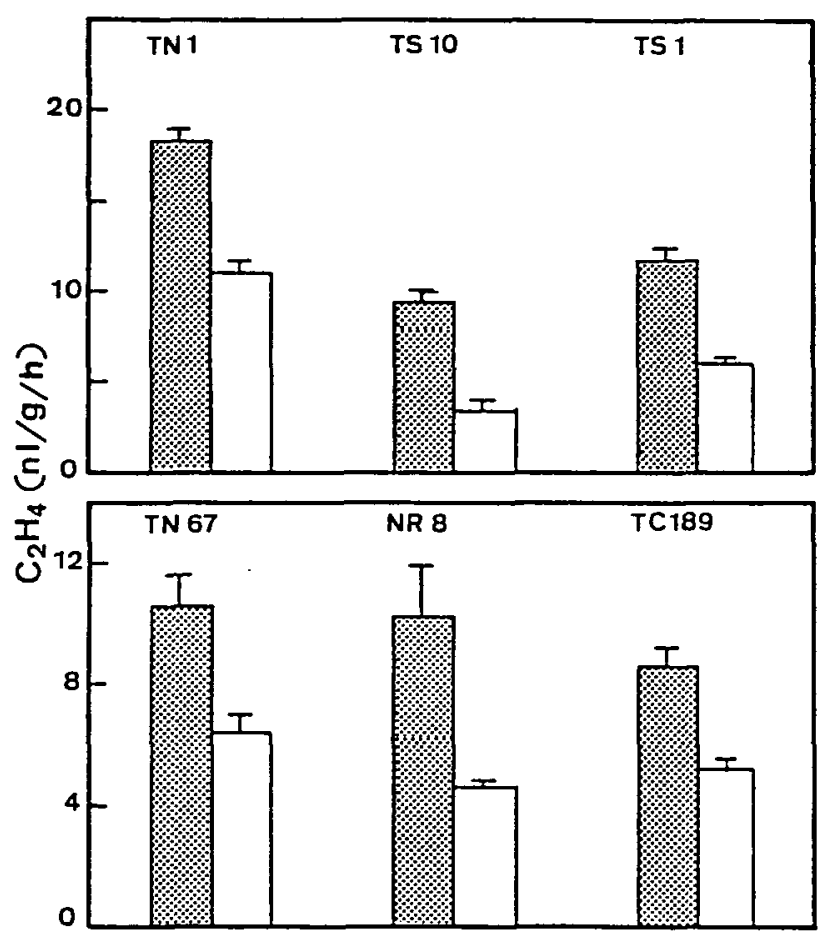

Fig. 6 Put-induced production of ethylene in segments of leaves from 6 rice cultivars. Cultivars in the upper panel are Indica varieties, whereas those in the lower panel are Japonica varieties. TN1, Taichung Native 1; TS10, Taichung Sen 10; TS1, Tai Sen 1; TN67, Tainung 67; NR8, Norin 8; TC189, Taichung 189. Segments of leaves were treated with either water (open columns) or $10 \mathrm{~mm}$ Put (shaded columns) in darkness. The production of ethylene was assayed $12 \mathrm{~h}$ after treatment. Bars indicate standard errors.

ined whether or not the conversion of ACC to ethylene is stimulated by polyamines in tobacco leaf discs. In contrast to the results obtained with detached rice leaves, polyamines significantly inhibited the conversion of ACC to ethylene in tobacco leaf discs (data not shown). Our results in this respect agree with those of Apelbaum et al. (1981) and indicate that the effects of polyamines on the production of ethylene in detached rice leaves differ from those in tobacco leaf discs.

\section{Discussion}

Polyamines have been reported to inhibit the production of ethylene in a number of plant tissues, including fruits, leaves, petals, and hypocotyls (Apelbaum 1981, Fuhrer et al. 1982, Ke and Romani 1988, Suttle 1981). In some instances, polyamines have been shown to have no detectable effect on the production of ethylene (Downs and Lovell 1986, Kramer and Wang 1990). Unexpectedly, we found that polyamines stimulate the production of ethylene in detached rice leaves under both light and dark conditions. The effects of polyamines on the promotion of the production of ethylene are unlikely to be specific for the particular rice cultivar used in this study, since polyamines also promoted the production of ethylene in detached leaves of five other cultivars of rice. The effects of polyamines on the production of ethylene seem to be complex. At present, it is impossible to propose a general explanation for the stimulation by polyamines of the production of ethylene in rice leaf tissues and the inhibition in other tissues and leaf tissues of other species. Since polyamines have been found to inhibit the production of ethylene in leaf tissues of oat, bean and tobacco (Apelbaum et al. 1981, Fuhrer et al. 1982), it is unlikely that polyamines stimulate the production of ethylene in monocots predominantly. Using internodes of submerged deep-water rice plants, Cohen and Kende (1986) found that enhanced rates of biosynthesis of ethylene and polyamines occurred in parallel, and one process did not inhibit the other. Their results seem to be consistent with ours.

One of the main questions in all hormone research is whether or not a particular hormone acts directly or indirectly on the process under examination. Although polyamines are regarded as members of a more loosely defined category, namely, that of plant growth regulators, rather than being considered to be actual hormone (Evans and Malmberg 1989), it is also of interest to determine whether they act directly or indirectly on the production of ethylene. This question was examined in the present study by following the time course of the effect of Put. Results from the present study clearly showed that Put induced a significant increase in the production of ethylene shortly (within $4 \mathrm{~h}$ ) after application. This observation suggests that Put stimulates the biosynthesis of ethylene directly. Since polyamines and ethylene have opposite effects on the senescence of detached rice leaves (Cheng and Kao 1983, Kao and Yang 1983), the polyamine-enhanced production of ethylene in detached rice leaves is unlikely to be mediated via mechanism associated with senescence. The finding that polyamines stimulate the production of ethylene suggests that the mechanism of retardation of senescence of detached rice leaves by polyamines does not involve the response to ehtylene.

Why is the extent of the stimulatory effect of polyamines on the production of ethylene quite small, usually less than two-fold? One possibility is that polyamines are not readily transported in rice leaf cells, as we reported previously (Cheng and Kao 1983). It should be noted that, in the present study, segments of unpeeled leaves were used.

The present investigation demonstrated that the increased production of ethylene in Put-treated leaves is regulated via promotion of the synthesis of $A C C$ and the conversion of ACC to ethylene. Evidence, though in- 
direct, is also provided to show that the observed increases in levels of ACC are mediated by increases in the activity of ACC synthase and in the level of the substrate (SAM) for ACC synthase. However, it is unclear whether the increase in the activity of ACC synthase in Put-treated leaves is regulated by the de novo synthesis of ACC synthase and/ or by the activation of preexisting ACC synthase.

Of particular interest is the finding that Put inhibits the activity of SAMDC. Since both ethylene and polyamines share SAM as a common precursor (Miyazaki and Yang 1987), our observations suggest that the increased levels of ethylene produced may be, at least in part, due to a redirection of SAM from the polyamine biosynthetic pathway as a result of some feedback-inhibition mechanism activated by the application of polyamines.

It is generally accepted that the conversion of ACC to ethylene is the final step in the biosynthesis of ethylene which involves a highly structured system that requires integrity of the cell membranes (Yang and Hoffman 1984). Polyamines have been shown to stabilize membranes (Smith 1985). Thus, in detached rice leaves, polyamines may enable cell membranes to retain their functions which, in turn, lead to the stimulation of the production of ethylene. The results of the examination of the effects of aging seem to support this hypothesis.

This work was supported by a research grant from the National Science Council of the Republic of China (NSC80-0409-B002-04).

\section{References}

Apelbaum, A., Burgoon, A. C., Anderson, J. D., Liberman, M., Ben-Arie, R. and Mattoo, A. K. (1981) Polyamines inhibit biosynthesis of ethylene in higher plant tissue and fruit protoplasts. Plant Physiol. 68: 453-456.

Chen, C. T., Chou, I. T. and Kao, C. H. (1990) Senescence of rice leaves $\mathrm{XX}$. Changes of proton secretion during senescence. Plant Sci. 66: 29-34.

Cheng, S. H. and Kao, C. H. (1983) Localized effect of polyamines on chlorophyll loss. Plant Cell Physiol. 24: 1463-1467.

Cohen, E. and Kende, H. (1986) The effect of submergence, ethylene and gibberellin on polyamines and their biosynthetic enzymes in deepwater-rice internodes. Planta 169: 498-504.

Downs, C. G. and Lovell, P. H. (1986) The effect of spermidine and putrescine on the senescence of cut carnations. Physiol. Plant. 66: 679-684.

Evans, P. T. and Malmberg, R. L. (1989) Do polyamines have roles in plant development? Annu. Rev. Plant Physiol. Plant Mol. Biol. 40: 235-269.

Fuhrer, J., Kaur-Sawhney, R., Shih, L. M. and Galston, A. W. (1982) Effects of exogenous 1,3-diaminopropane and spermidine on senescence of oat leaves II. Inhibition of ethylene biosynthesis and possible mode of action. Plant Physiol. 70: 1597-1600.

Kao, C. H. and Yang, S. F. (1982) Light inhibition of the conversion of 1-aminocyclopropane-1-carboxylic acid to ethylene in leaves is mediated through carbon dioxide. Planta 155: 261266.

Kao, C. H. and Yang, S. F. (1983) Role of ethylene in the senescence of detached rice leaves. Plant Physiol. 73: 881-885.

Kaur-Sawhney, R., Shih, L. M., Flores, H.E. and Galston, A. W. (1982) Relationship of polyamine synthesis and titer to aging and senescence in oat leaves. Plant Physiol. 69: 405-410.

Ke, D. and Romani, R. J. (1988) Effects of spermidine on ethylene production and the senescence of suspension-cultured pear fruit cells. Plant Physiol. Biochem. 26: 109-116.

Kramer, G. F. and Wang, C. Y. (1990) Inhibition of softening of apples by postharvest polyamine infiltration. Plant Physiol. 93: $\mathrm{S}-442$.

Lowry, O. H., Rosebrough, N. J., Farr, S. L. and Randall, R. J. (1951) Protein measurement with the Folin phenol reagent. $J$. Biol. Chem. 193: 265-275.

Metraux, J. P. and Kende, H. (1984) The cellular basis of the elongation response in submerged deep-water rice. Planta 160: 73-77.

Miyazaki, J. H. and Yang, S. F. (1987) The methionine salvage pathway in relation to polyamine biosynthesis. Physiol. Plant. 69: 366-370.

Shih, L. M., Kaur-Sawhney, R., Fuhrer, J., Samanta, S. and Galston, A. W. (1982) Effects of exogenous 1,3-diaminopropane and spermidine on senescence of oat leaves 1 . Inhibition of protease activity, ethylene production, and chlorophyll loss as related to polyamine content. Plant Physiol. 70: 1592-1596.

Smith, T. A. (1985) Polyamines. Annu. Rev. Plant Physiol. 26: 117-143.

Suttle, J. C. (1981) Effect of polyamines on ethylene production. Phytochemistry 20: 1477-1480.

Yang, S. F, and Hoffman, N.E. (1984) Ethylene biosynthesis and its regulation in higher plants. Annu. Rev. Plant Physiol. 35: 155-189.

(Received February 27, 1991; Accepted June 5, 1991) 
